\title{
Boxy/peanut and discy bulges: formation, evolution and properties
}

\author{
E. Athanassoula \\ Laboratoire d'Astrophysique de Marseille, Observatoire Astronomique de Marseille Provence, \\ 2 place Le Verrier, 13248 Marseille cedex 04, France
}

\begin{abstract}
The class 'bulges' contains objects with very different formation and evolution paths and very different properties. I review two types of 'bulges', the boxy/peanut bulges (B/Ps) and the discy bulges. The former are parts of bars seen edge-on, have their origin in vertical instabilities of the disc and are somewhat shorter in extent than bars. Their stellar population is similar to that of the inner part of the disc from which they formed. Discy bulges have a disc-like outline, i.e., seen face-on they are circular or oval and seen edge-on they are thin. Their extent is of the order of 5 times smaller than that of the boxy/peanut bulges. They form from the inflow of mainly gaseous material to the centre of the galaxy and from subsequent star formation. They thus contain a lot of young stars and gas. Bulges of different types often coexist in the same galaxy. I review the main known results on these two types of bulges and present new simulation results.

B/Ps form about 1Gyr after the bar, via a vertical buckling. At that time the bar strength decreases, its inner part becomes thicker - forming the peanut or boxy shape - and the ratio $\sigma_{z}^{2} / \sigma_{r}^{2}$ increases. A second buckling episode is seen in simulations with strong bars, also accompanied by a thickening of the peanut and a weakening of the bar. The properties of the B/Ps correlate strongly with those of the bar: stronger bars have stronger peanuts, a more flat-topped vertical density distribution and have experienced more bucklings.

I also present simulations of disc galaxy formation, which include the formation of a discy bulge. Decomposition of their radial density profile into an exponential disc and a Sérsic bulge gives realistic values for the disc and bulge scale-lengths and mass ratios, and a Sérsic shape index of the order of 1 .

It is thus clear that classical bulges, $\mathrm{B} / \mathrm{P}$ bulges and discy bulges are three distinct classes of objects and that lumping them together can lead to confusion. To avoid this, the two latter could be called $\mathrm{B} / \mathrm{P}$ features and inner discs, respectively.
\end{abstract}

Keywords. galaxies: bulges, galaxies: evolution, galaxies: formation, galaxies: kinematics and dynamics, stellar dynamics, methods: N-body simulations

\section{Introduction}

What is a bulge? Three different definitions have been used so far, based on morphology, photometry, or kinematics, respectively. According to the morphological definition, a bulge is the component of a disc galaxy that swells out of the central part of a disc viewed edge-on. Based on photometry, a bulge is the extra light in the central part of the galaxy, over and above the exponential profile fitting the remaining (non central) part of the disc. The third definition is based on kinematics, and in particular on the value of $V / \sigma$, or, more specifically, on the location of the object on the $(V / \sigma$, ellipticity) diagram (often referred to as the Binney diagram (Binney 1978, 2005)). These three definitions are compared and discussed in Athanassoula \& Martinez-Valpuesta (2007).

The lack of a single, clear-cut definition of a bulge, although historically understandable, has led to considerable confusion and to the fact that bulges are an inhomogeneous class of objects. For this reason, Kormendy (1993; see also Kormendy \& 
Kennicutt 2004, hereafter KK04) distinguished classical bulges from pseudo-bulges. However, pseudo-bulges by themselves are also an inhomogeneous class of objects, as argued by Athanassoula (2005a, hereafter A05), who distinguishes three types of objects which are, according to the above definitions, classified as bulges. Classical bulges are formed by gravitational collapse or hierarchical merging of smaller objects and corresponding dissipative gas processes. Their morphological, photometrical and kinematical properties are similar to those of ellipticals. They are discussed extensively in other papers in these proceedings and are not the subject of this review. The two other types of bulges are boxy/peanut bulges $(\mathrm{B} / \mathrm{P})$, and discy bulges, which will be discussed here. As stressed in A05, different types of bulges often co-exist and it is possible to find all three types of bulges in the same simulation, or in the same galaxy.

\section{Boxy/peanut bulges}

Viewed edge-on, disc galaxies often have a central component which swells out of the disc and whose outline is not elliptical, but has a boxy, or peanut, or even ' $\mathrm{X}$ ' shape. Due to the morphological definition of a bulge, such components have been called bulges, or, more specifically, boxy/peanut bulges (B/Ps).

The formation of $\mathrm{B} / \mathrm{Ps}$ has been witnessed in a large number of numerical simulations (Combes \& Sanders 1981; Combes et al. 1990; Raha et al. 1991; Athanassoula \& Misiriotis 2002, hereafter AM02; Athanassoula 2003, hereafter A03; A05; O’Neil \& Dubinski 2003; Debattista et al. 2004; Martinez-Valpuesta \& Shlosman 2004; Debattista et al. 2006; Martinez-Valpuesta, Shlosman \& Heller 2006, etc). It is linked to the vertical instability of parts of the main family of periodic orbits constituting the bar, widely known as the $\mathrm{x}_{1}$ family (Binney 1981; Pfenniger 1984; Skokos, Patsis \& Athanassoula 2002; Patsis, Skokos \& Athanassoula 2002). The stability of the $\mathrm{x}_{1}$ family can be followed from the corresponding stability diagram (see e.g. figures 3 and 4 of Skokos et al. 2002) which shows that, at the positions where the $\mathrm{x}_{1}$ becomes unstable, other families bifurcate. These are linked to the $n: 1$ vertical resonances and extend well outside the disc equatorial plane. As shown by Patsis et al. (2002), some of them are very good building blocks for the formation of peanuts, because they are stable and because their orbits have the right shape, extent and location. Studies of these orbits reproduced many of the B/P properties and helped explaining crucial aspects of $\mathrm{B} / \mathrm{P}$ formation and evolution. For example, an analysis of the orbital families that constitute peanuts predicts that B/Ps should be shorter than bars. This is indeed found to be the case both in $N$-body simulations and in real galaxies (Lütticke, Dettmar \& Pohlen 2000; A05; Athanassoula \& Beaton 2006).

\subsection{Time evolution}

The time evolution of the bar, of the buckling and of the peanut strengths are plotted in Fig. 1 for a simulation which develops a strong bar. The time is given in Gyrs, using the calibration proposed in AM02. The initially unbarred disc forms a bar roughly between times 3 and 4 Gyrs (lower panel). I define as bar formation time the time at which the bar-growth is maximum (i.e. when the slope of the bar strength as a function of time is maximum) and indicate it by the first vertical line in Fig. 1. The bar strength reaches a maximum at a time indicated by the second vertical line, and then decreases considerably over $\sim 1$ Gyr. The time at which the bar amplitude decrease is maximum is given by the third vertical line. Subsequently, the bar strength reaches a minimum, at a time shown by the fourth vertical line, and then starts increasing again at a rate much slower than that during bar formation.

The upper panel shows the buckling strength, i.e. the vertical asymmetry as a function of time. The disc is vertically symmetric before and during bar formation and the first 


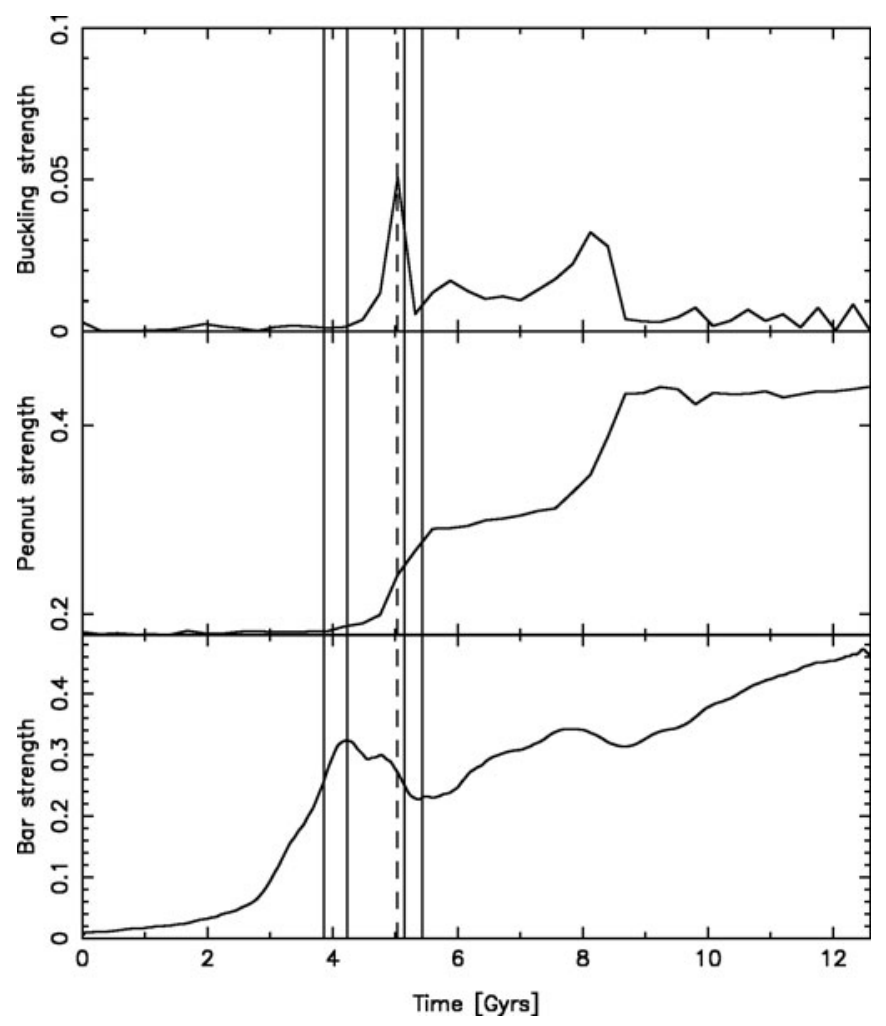

Figure 1. Time evolution of three peanut-, or bar-related quantities, namely the buckling strength (i.e. the vertical asymmetry; upper panel), the peanut strength (i.e. its vertical extent; middle panel) and the bar strength (lower panel). The solid vertical lines mark characteristic times linked to bar formation and evolution. From left to right, these are the bar formation time, the maximum amplitude time, the bar decay time and the bar minimum amplitude time (see text). The vertical dashed line marks the time of the buckling.

indications of asymmetry occur only after the bar amplitude has reached a maximum. The asymmetry then grows very abruptly to a strong, clear peak and then drops equally abruptly. The time of the buckling (dashed vertical line) is given by the peak of this curve and is very clearly defined. It is important to note that, to within the measuring errors, it coincides with the time of bar decay (third vertical line). This is not accidental. I verified it for a very large number of simulations and thus can establish the link between the buckling episode and the decay of the bar strength (Raha et al. 1991; MartinezValpuesta \& Shlosman 2004).

The middle panel shows the strength of the peanut, i.e. its vertical extent, again as a function of time. This quantity grows abruptly after the bar has reached its maximum amplitude and during the time of the buckling. This abrupt growth is followed by a much slower increase over a longer period of time. Taken together, the three panels of Fig. 1 show that the bar forms vertically thin, and only after it has reached a maximum strength does the buckling phase occur. During the buckling time the bar strength decreases significantly, while the the $\mathrm{B} / \mathrm{P}$ strength increases. The time intervals during which bar formation, peanut formation, or buckling occur are all three rather short, of the order of a Gyr, and they are followed by a longer stretch of time during which the bar and B/P evolve much slower. This later evolution is often referred to as secular evolution.

This particular simulation has a second, weaker buckling episode shortly after 8 Gyrs. This occurs very often in simulations developing strong bars and was discussed first by 
Athanassoula (2005b) and Martinez-Valpuesta et al. (2006). It is seen clearly in all three panels and has characteristics similar to those of the first buckling.

\subsection{Peanut formation and collective effects}

As already discussed, orbital structure theory explains $\mathrm{B} / \mathrm{P}$ formation by the vertical instabilities of the main family of bar-supporting periodic orbits. An alternative approach explains the buckling and the peanut formation as due to the bending, or fire-hose, instability, studied analytically in the linear regime (Toomre 1966; Araki 1985). These studies assign a critical value to the ratio $R_{\sigma}=\sigma_{z}^{2} / \sigma_{r}^{2}$ igniting the onset of the instability, which is around 0.1. A number of simulations, however, have shown that the vertical instability sets in at much larger values of $R_{\sigma}$ (e.g. Merritt \& Sellwood 1994; Sotnikova \& Rodionov 2003).

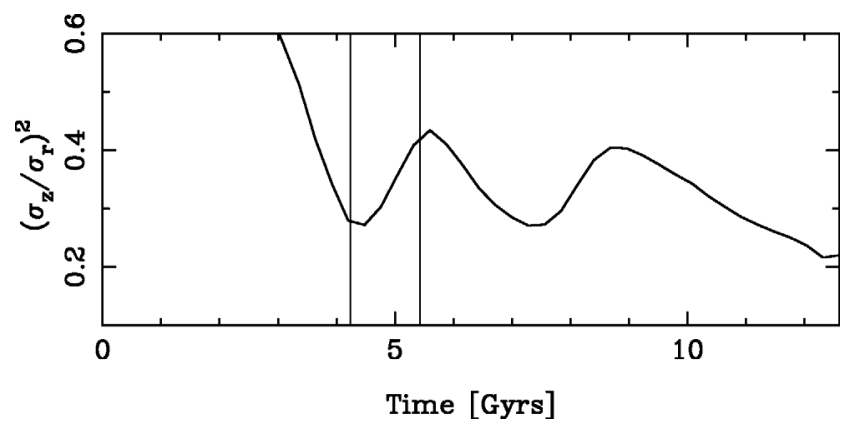

Figure 2. Time evolution of the ratio $R_{\sigma}=\sigma_{z}^{2} / \sigma_{r}^{2}$. The thin vertical lines mark two characteristic times linked to bar formation and evolution. From left to right, these are the bar maximum and minimum amplitude times, corresponding to the first buckling episode (see Sect. 2.1 and Fig. 1).

To test this hypothesis, I calculate the radial and $z$ components of the disc velocity dispersion as a function of radius (averaging over azimuth and height). I then find the minimum value of their ratio $R_{\sigma}$ and plot its time evolution in Fig. 2. The thin vertical lines mark two characteristic times linked to bar formation and evolution - namely the bar maximum and minimum amplitude times - as found from Fig. 1. Their location is clearly linked to changes in behaviour of $R_{\sigma}$. This, however, does not necessarily imply that the changes in $R_{\sigma}$ are the cause of the buckling, but can also be seen as its consequence. Indeed, as the bar forms $\sigma_{r}$ increases drastically, so that $R_{\sigma}$ decreases. Then the bar amplitude reaches its maximum and starts decreasing, while the peanut starts forming. During this time, $\sigma_{r}$ decreases while $\sigma_{z}$ increases. As a result, the ratio $R_{\sigma}$ reaches a minimum when the bar amplitude is maximum and then increases again, as is indeed seen in Fig. 2. Then the bar amplitude reaches a minimum, which corresponds to a minimum of $\sigma_{r}$ and therefore to a maximum of $R_{\sigma}$. This is followed by a slower decrease of $R_{\sigma}$, which is stopped by the second buckling episode. The value of $R_{\sigma}$ at which this instability sets in is much less extreme than that predicted by the above mentioned analytical works, but is in good agreement with other $N$-body simulations.

More work is necessary before we fully understand the respective roles of the orbital structure results and of the velocity anisotropy effects on the formation and evolution of $\mathrm{B} / \mathrm{P}$ structures. Both explain part of the story, but many aspects of their interplay are still unclear. Orbital structure results tell us whether the appropriate building blocks are available, or not, and this is essential, since the lack of such building blocks prohibits the formation of a given structure. Furthermore, studies of the properties of the buildingblock orbits are essential for understanding the properties of the $\mathrm{B} / \mathrm{P}$ structures. Orbital 
structure theory, however, can not tell us how much matter is trapped around a given orbit or family. Furthermore, it is necessary to group all these building blocks into one coherent, self-consistent unit and here collective effects are essential. Like orbital structure, they also can set limits on the formation of $\mathrm{B} / \mathrm{P}$ structures, as well as give information on their properties. The respective input from the two methods will be discussed further elsewhere.

\subsection{Comparison with observations}

The fact that B/Ps are just parts of bars seen edge-on was not immediately accepted (see e.g. Kormendy 1993). The main arguments against it were, however, refuted in A05, with the help of orbital structure results. Furthermore, considerable observational evidence argues in its favour, particularly detailed comparisons between observations and simulations.

Radial density profiles from simulations, taken along slits on, or parallel to, the equatorial plane when the galaxy is seen edge-on (AM02; A05) have the same characteristic signatures as the corresponding radial light profiles (Lütticke, Dettmar \& Pohlen 2000; Bureau et al. 2006). Similarly, density profiles along cuts perpendicular to the equatorial plane (AM02; A05) show similar characteristics to analogous observed light profiles (Aronica et al. 2003 and this volume). Further tests come from comparisons of median filtered images of $\mathrm{B} / \mathrm{P}$ systems (Bureau et al. 2006) to similar images of $N$-body bars (A05). These show the same types of characteristic features, namely four extensions out of the equatorial plane, which form an X-like shape, except that the four extensions do not necessarily cross the centre. Another common feature is maxima of the density along the equatorial plane, away from the centre and diametrically opposite. Starting from the centre of the galaxy and going outwards along the equatorial plane, the projected surface density first drops, then increases again to reach a local maximum and then decreases again to the edge of the disc.

Considerable evidence was also accumulated using kinematical observations. Cylindrical rotation, witnessed in a number of B/P galaxies (KK04 and references therein), is also seen in velocity fields of strong $N$-body bars viewed edge-on (Combes et al. 1990; AM02). Emission line spectroscopy of boxy/peanut galaxies (Kuijken \& Merrifield 1995; Bureau \& Freeman1999; Merrifield \& Kuijken 1999) shows that their major axis position velocity diagrams (PVDs) have a number of interesting features, well reproduced by gas flow simulations (Athanassoula \& Bureau 1999). In particular, the shocks along the leading edges of the bar and the corresponding inflow lead to a characteristic gap in the PVDs, between the signature of the nuclear spiral (whenever existent) and the signature of the disc.

Comparison of long-slit absorption line spectra (Chung \& Bureau 2004) of galaxies with $\mathrm{B} / \mathrm{Ps}$ to similar 'observations' of $N$-body bars viewed edge-on reveals that the two have the same characteristic features (Bureau \& Athanassoula 2005). The integrated light along the slit (equivalent to a major-axis light profile) has a quasi-exponential central peak and a plateau at intermediate radii, followed by a steep drop. The rotation curve $(V(r))$ has a characteristic double hump. The velocity dispersion has a central peak, which in the centre-most part may be rather flat or may even have a central minimum. At intermediate radii there can be a plateau which sometimes ends on either side with a shallow maximum before dropping steeply at larger radii. $h_{3}$ (i.e. the coefficient of the third order term in a Gauss-Hermite expansion of the line of sight velocity distribution) correlates with $V$ over most of the bar length, contrary to what is expected for a fast rotating disc. All these features are spatially correlated and are seen, more or less strongly, both in the observations and in the simulations (Bureau \& Athanassoula 2005). 

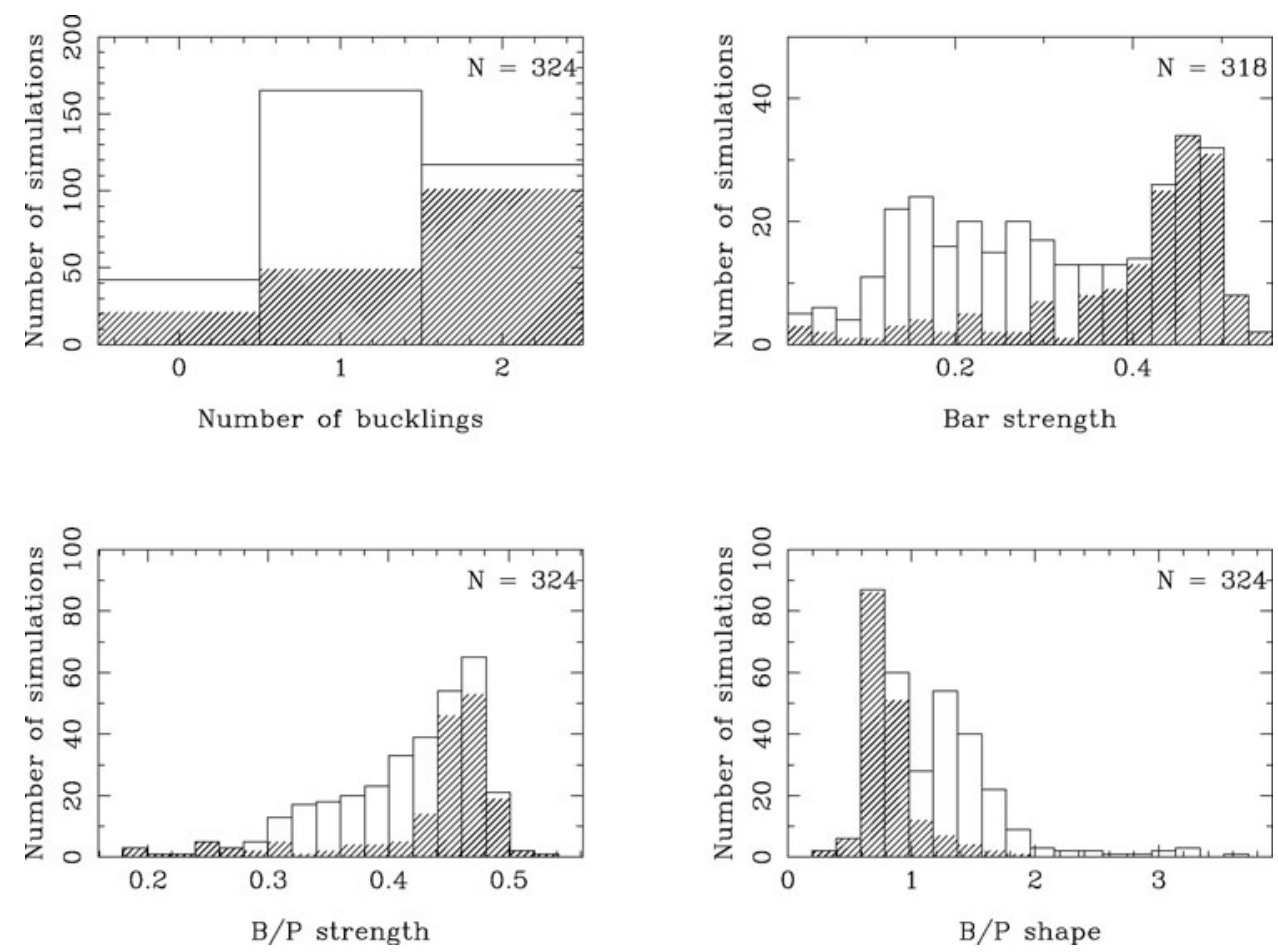

Figure 3. Histograms of number of bucklings, bar strength, B/P strength and B/P shape for a sample of fully self-consistent high-resolution simulations. The histograms include simulations with halo cores of all sizes and the hatched areas includes only simulations with small halo cores.

In collaboration with Martinez-Valpuesta, I made an extensive statistical study of a few hundred simulations which I had run for different purposes (AM02; A03 etc). We measured the strength of the peanut (from its thickness) and its shape (from the shape of the density profile on cuts perpendicular to the equatorial plane) and found that both correlate well with the bar strength. Thus, stronger bars have stronger peanuts and more flat-topped vertical density profiles. I also find that the type of halo plays a major role in determining the properties of the B/P. Fig. 3 shows histograms of the number of bucklings that have occurred, of the bar strength, of the $\mathrm{B} / \mathrm{P}$ strength and of the $\mathrm{B} / \mathrm{P}$ shape, distinguishing between simulations with small halo cores and simulations with large halo cores. The two populations are indeed very different. Simulations with small halo cores have stronger bars, stronger peanuts, more flat-topped vertical density profiles and have experienced more bucklings than simulations with large cores. The few simulations with small cores which have weak bars, weak B/Ps and did not buckle have either a very hot halo or a very hot disc. Simulations with cuspy haloes (not shown here) have yet weaker B/Ps and smaller number of bucklings and will be discussed elsewhere.

The above can be explained by the fact that the halo plays a major role in determining the properties of the bar (AM02; A03). Athanassoula (2002, hereafter A02) showed that angular momentum is primarily emitted by near-resonant material in the bar region and absorbed by near-resonant material in the outer disc and, particularly, in the halo, while A03 showed that bars grow stronger when more angular momentum is exchanged within the galaxy. Furthermore, as shown in AM02 and as explained in A02 and A03, 
the size of the halo core strongly influences the bar evolution. Haloes with a small core have a lot of mass in the inner regions and thus, provided their velocity dispersion is not too high, can provide substantial angular momentum sinks and lead to considerable angular momentum exchange between the near-resonant particles in the bar region and the near-resonant particles in the halo. Such models grow strong bars (long, thin and massive) with rectangular-like isodensities (AM02). Viewed side-on (i.e. edge-on with the line-of-sight along the bar minor axis) they exhibit a strong peanut, or even X-like shape. If, however, the velocity dispersion in the disc and/or halo is too high, the angular momentum exchange is hindered and the bar and peanut will be weak (A03). Haloes with large cores have considerably less material in the inner parts and are thus capable of less angular momentum exchange. Bars grown in such environments are less strong, have elliptical-like isodensities when viewed face-on and boxy-like when viewed side-on. All these considerations explain the results found in Fig. 3, namely the difference between the histograms for simulations with small halo cores and simulations with large halo cores. They also explain the weak bars and B/Ps found in some simulations with small halo cores.

\section{Disc-like bulges}

Disc-like bulges form from inflow of (mainly) gas material to the centre of the galaxy and subsequent star formation. This inflow is due to the torques exerted by a nonaxisymmetric component, usually a large-scale bar, as witnessed in hydrodynamic simulations (e.g. Athanassoula 1992; Friedli \& Benz 1993; Heller \& Shlosman 1994; Wada \& Habe 1995). The high density of the gas accumulated in the inner regions triggers very strong star formation. Such inflow, however, is also seen in $N$-body simulations (AM02; Valenzuela \& Klypin 2003), which represent the old stellar population. Thus, this inner region is not only a region of increased density for the gas and the young stars, but also for the older stellar populations. This should lead to the formation of an inner, central component of disc-like shape, whose extent is of the order of a $\mathrm{kpc}$ and which is constituted mainly of gas and young stars, but also of older stars. This was named disc-like bulge, or, for short, discy bulge in A05 and is often observed in disc galaxies. Due to its disc-like shape, it often has spirals or inner bars (KK04 and references therein). It stands out very clearly in radial photometric profiles, whose decomposition shows it is well represented by a Sérsic law (Sérsic 1968). Contrary to classical bulges, however, it does not swell out of the galactic plane. This is not the only difference between disc-like and classical bulges. Disc-like bulges have a Sérsic index of the order of 1, i.e. much smaller than the values found for classical bulges (KK04 and references therein). They also have different kinematics, like that of discs, a higher fraction of young stars and a higher gas content. A lot of data on such bulges has been collected over the last few years, but still much work, particularly theoretical, is necessary before we fully understand these objects.

In order to describe adequately the formation and evolution of disc-like bulges, simulations should include gas, star formation and feedback, all in a realistic way. It would, furthermore, be preferable if they started from cosmological or cosmologically-motivated initial conditions, since the properties of pre-existing discs may influence the properties of the disc-like bulges. I will briefly describe here results from simulations following this outline (Athanassoula, Heller \& Shlosman, in preparation). For information on the numerical techniques used in these simulations and an initial discussion of some of the results see Heller, Shlosman \& Athanassoula (2007a) and (2007b).

Several non-axisymmetric components - such as a triaxial halo, oval disc, inner and outer bar - form during these simulations. Their interactions give very interesting 

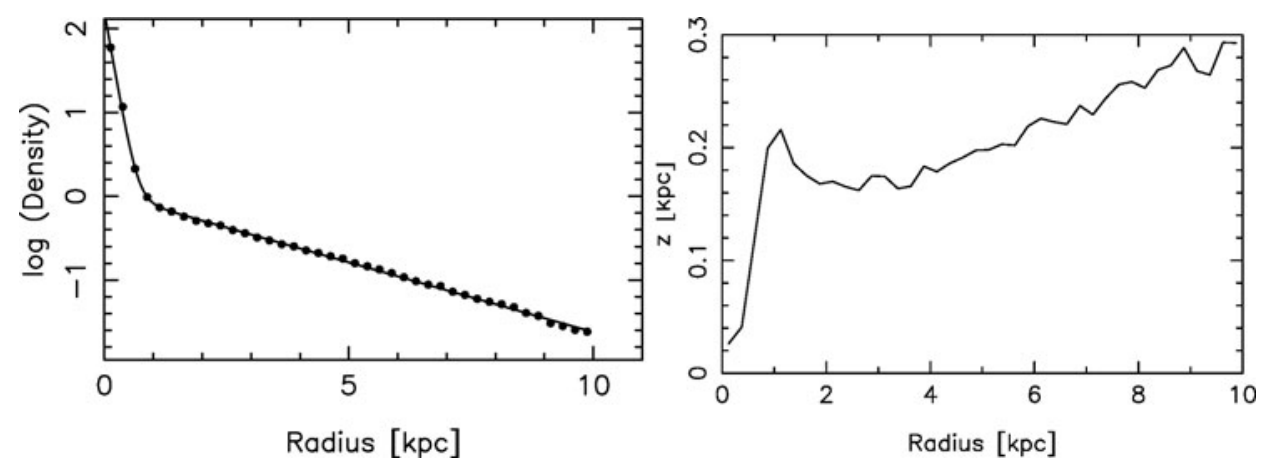

Figure 4. Properties of a simulated disc-like bulge. Left : Radial projected density profile in arbitrary units. Radii are measured in kpc. The dots give the simulation results and the straight line the fit by an exponential disc and a Sérsic component. Right : Measure of the vertical height of the material near the equatorial plane (see text), as a function of radius, measured in kpc.

dynamical phenomena (Heller et al. 2007a; b), while they induce considerable inflow and gaseous high density inner discs. As in the sketchy outline in the beginning of this section, the high gas concentration in the central area triggers considerable star formation, resulting in a disc-like central, high-density object, which, seen face-on, is often somewhat oval. It has many properties similar to those of discy-bulges. For example, it has, in many cases, sub-structures, like an inner bar. In order to further assess the properties of the disc galaxy formed in these simulations and to better establish the link with disc-like bulges, I chose a characteristic specific snapshot, i.e. a characteristic specific simulation and time, and examine its mass distribution in order to compare best with the observed light distribution in galaxies. An analysis of the kinematics, together with a statistical treatment, including other times and other simulations, will be given elsewhere. The radial projected surface density profile of the snapshot under consideration is given in Fig. 4, together with a fit by an exponential disc and a Sérsic component. Note that the fit is excellent, all the way to the outer parts of the disc, roughly at $10 \mathrm{kpc}$. In this example, the disc scale-length is $\sim 2.7 \mathrm{kpc}$, i.e. very realistic, while the Sérsic index is $\sim 1$, in good agreement with observed discy bulges.

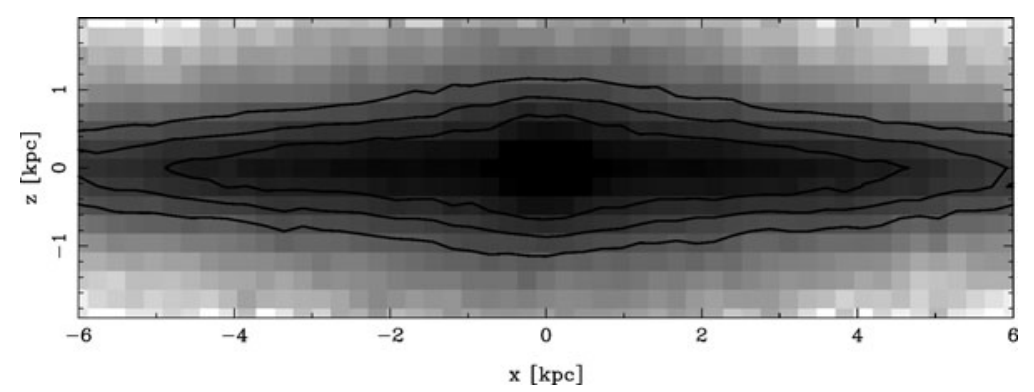

Figure 5. Edge-on view of the stellar component of the simulation with a disc and a disc-like bulge. The projected density is given by grey-scale and also by five isocontours whose level is picked so as to show best the features under consideration.

Fig. 5 shows the snapshot seen edge-on. The three outer isodensity curves show clearly that the shape and aspect ratio of the disc component is very realistic. The two innermost contours (within $1 \mathrm{kpc}$ ) reveal the existence of a small, central, disc-like object, the vertical height of which we need to quantify. Measuring the average thickness would not be useful, since this is due to both the external big disc and the small inner component, so 
I proceeded differently. I divided the 'stars' in the snapshot into circular annuli, according to their distance from the centre and, in each annulus, sorted them as a function of their distance from the equatorial plane $(|z|)$. Since, statistically, the 'stars' in the disc-like inner component will have smaller $|z|$ values than the ones in the outer disc, I plot in Fig. 4 the $|z|$ component of the 'star' with rank $0.3 N_{a n}$, where $N_{a n}$ is the total number of 'stars' in the annulus. This shows a deep minimum in the central region, as one would expect due to the existence of an inner disc with a shorter vertical extent than the outer one. It also shows that the region where the inner disc is contributing significantly is of the order of $1 \mathrm{kpc}$, in good agreement with the radial density profile (Fig. 4). Finally, the aspect ratio of the inner and the outer discs are similar.

To summarise, in our fiducial simulation, as well as in several others, we witness inflow of mainly gas material to the central regions and strong subsequent star formation. Thus, an inner disc is formed, composed of both stars and gas. Its radial extent is of the order of a kpc and its vertical extent much smaller than that of the outer disc. This disc can harbour spiral structure, or an inner bar. Its contribution to the radial projected density profile is well fitted by a Sérsic law with Sérsic index $\sim 1$. It is thus very likely that this simulation describes correctly the formation of discy-like bulges in galaxies.

\section{Summary and discussion}

I briefly reviewed the formation, evolution and properties of boxy/peanut bulges and of disc-like bulges. These two types of objects have very different formation and evolutionary histories and very different properties. B/Ps form from vertical instabilities and their building blocks are the $3 \mathrm{D}$ families associated with the $3 \mathrm{D}$ bifurcations of the $\mathrm{x}_{1}$ family. Discy bulges form from the inflow of (mainly) gas material and from the ensuing enhanced star formation. Thus B/Ps are mainly constituted of inner disc stars, while the discy bulges have a very large contribution from gas and young stars. Since the formation of discy bulges relies on the gas inflow, it is expected that they will be found mainly in late type disc galaxies, as is indeed the case. The face-on extent of the B/Ps is of the order of five times larger than that of the discy bulges and, seen edge-on, they extend well outside the equatorial plane, while the discy bulges are thin. Their kinematics and their contribution to the radial photometric profiles are different from those of discy bulges. Thus, one should clearly distinguish between B/Ps and discy-bulges and not lump together them in a single category.

Once this has become clear, one may also wish to revise the existent nomenclature in order to avoid some of the present confusion. Boxy/peanut bulges could be called boxy/peanut features (or structures), or simply peanuts, as proposed in A05. This would make it clearer that they are just a part or a feature of the bar and not an independent entity. Similarly, discy bulges could be simply called inner discs. Then the name 'bulge' would be reserved for classical bulges. This change, however, will also necessitate changing the bulge definitions described in Sect. 1.

\section{Acknowledgements}

I thank my collaborators, A. Bosma, A. Aguerri, C. Heller, I. Martinez-Valpuesta and I. Shlosman, for interesting and fruitful discussions. This work was partially supported by grant ANR-06-BLAN-0172 and by the Grüber foundation.

\section{References}

Araki, S. 1985 PhD thesis, MIT 
Aronica, G., Athanassoula, E., Bureau, M., Bosma, A., Dettmar, R. -J., Vergani, D., \& Pohlen, M. 2003, Ap\&SS, 284, 753

Athanassoula, E. 1992, MNRAS, 259, 345

Athanassoula, E. 2002, ApJL, 569, 83 (A02)

Athanassoula, E. 2003, MNRAS, 341, 1179 (A03)

Athanassoula, E. 2005a, MNRAS, 358, 1477 (A05)

Athanassoula, E. 2005b, in Planetary Nebulae as Astronomical Tools, eds. R. Szczerba, G. Stasińska, \& S. K. Górny, AIP Conf. Proc. 804, Melville, New York, 333

Athanassoula, E., Beaton, R. L. 2006, MNRAS, 370, 1499

Athanassoula, E. \& Bureau, M. 1999, ApJ, 522, 699

Athanassoula, E., Misiriotis, A. 2002, MNRAS, 330, 35 (AM02)

Athanassoula, E., Martinez-Valpuesta 2007, in Pathways through an eclectic Universe, eds. J. H. Knapen, T. J. Mahoney, and A. Vazdekis, ASP Conf. Ser., 2007, in press and astro$\mathrm{ph} / 0710.1518$

Binney, J. 1978, MNRAS, 183, 501

Binney, J. 1981, MNRAS, 196, 455

Binney, J. 2005, MNRAS, 363, 937

Bureau, M., Aronica, G., Athanassoula, E., Dettmar, R.-J., Bosma, A., \& Freeman, K. C. 2006, MNRAS, 370, 753

Bureau, M. \& Athanassoula, E. 2005, ApJ, 626, 159

Bureau, M. \& Freeman, K. C. 1999, AJ, 118, 126

Chung, A. \& Bureau, M. 2004, AJ, 127, 3192

Combes, F. \& Sanders, R. H. 1981, A\&A, 96, 164

Combes, F., Debbasch, F., Friedli, D., \& Pfenniger, D. 1990, A\&A, 233, 82

Debattista, V. P., Carollo, M., Mayer, L., \& Moore, B. 2004, ApJ, 604, L93

Debattista, V. P., Carollo, M., Mayer, L. Moore, B., Wadsley, J., \& Quinn, T. 2006, ApJ, 645, 209

Friedli, D., \& Benz, W. 1993, A\&A, 268, 65

Heller, C. H. \& Shlosman, I. 1994, ApJ, 424, 84

Heller, C.H., Shlosman, I. \& Athanassoula, E. 2007a, ApJ, 657, L65

Heller, C. H., Shlosman, I., \& Athanassoula, E. 2007b, ApJ, 671, 226

Kormendy, J. 1993, in Galactic Bulges, eds. H. Dejonghe and H. J. Habing, Kluwer Academic Publ., IAU Symposium 153, 209

Kormendy, J., \& Kennicutt, R. C. 2004, ARA\&A, 42, 603 (KK04)

Kuijken, K. \& Merrifield, M. R. 1995, ApJ, 443, L13

Lütticke, R., Dettmar, R.-J. \& Pohlen, M. 2000, A\&A, 362, 435

Martinez-Valpuesta, I. \& Shlosman, I. 2004, ApJ, 613, 29

Martinez-Valpuesta, I., Shlosman, I., \& Heller, C. 2006, ApJ, 637, 214

Merrifield, M. R. \& Kuijken, K. 1999, A\&A, 345, L47

Merritt, D. \& Sellwood, J. A. 1994, ApJ, 425, 551

O'Neill, J. K. \& Dubinski, J. 2003, MNRAS, 346, 251

Patsis, P., Skokos, Ch., Athanassoula, E. 2002, MNRAS, 337, 578

Pfenniger, D. 1984, A\&A, 134, 373

Raha, N., Sellwood, J. A., James, R. A., \& Kahn, F. D. 1991, Nature, 352, 411

Sérsic, J. 1968, Atlas de Galaxias Australes, Obs. Astron. Cordoba

Skokos, H., Patsis, P. \& Athanassoula, E. 2002, MNRAS, 333, 847

Sotnikova, N. Ya. \& Rodionov, S. A. 2003, AstL, 29, 321

Toomre, A. 1966, Geophys. Fluid Dyn., N66-46, 111

Valenzuela, O., Klypin 2003, MNRAS, 345, 406

Wada, K. \& Habe, A. 1995, MNRAS, 277, 433 